\title{
Pseudounitary operators and pseudounitary quantum dynamics
}

Ali Mostafazadeh

Citation: Journal of Mathematical Physics 45, 932 (2004); doi: 10.1063/1.1646448

View online: http://dx.doi.org/10.1063/1.1646448

View Table of Contents: http://aip.scitation.org/toc/jmp/45/3

Published by the American Institute of Physics

\section{Articles you may be interested in}

Pseudo-Hermiticity versus PT symmetry: The necessary condition for the reality of the spectrum of a nonHermitian Hamiltonian

Journal of Mathematical Physics 43, 205 (2001); 10.1063/1.1418246

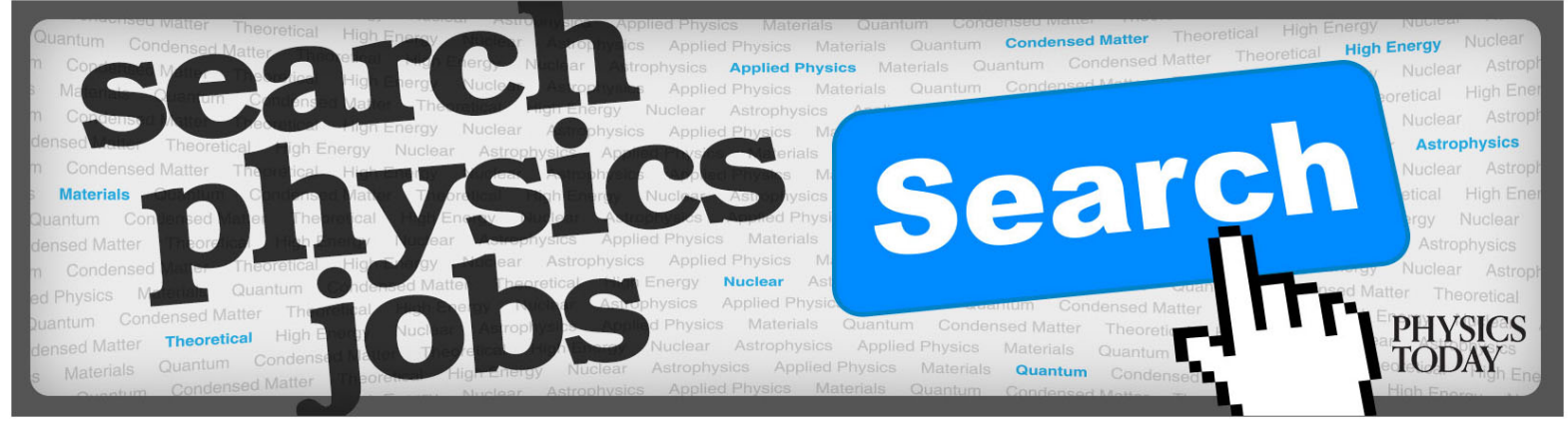




\title{
Pseudounitary operators and pseudounitary quantum dynamics
}

\author{
Ali Mostafazadeh ${ }^{\text {a) }}$ \\ Department of Mathematics, Koç University, Rumelifeneri Yolu, 34450 Sariyer, Istanbul, \\ Turkey
}

(Received 15 May 2003; accepted 15 December 2003)

\begin{abstract}
We consider pseudounitary quantum systems and discuss various properties of pseudounitary operators. In particular we prove a characterization theorem for block-diagonalizable pseudounitary operators with finite-dimensional diagonal blocks. Furthermore, we show that every pseudounitary matrix is the exponential of $i=\sqrt{-1}$ times a pseudo-Hermitian matrix, and determine the structure of the Lie groups consisting of pseudounitary matrices. In particular, we present a thorough treatment of $2 \times 2$ pseudounitary matrices and discuss an example of a quantum system with a $2 \times 2$ pseudounitary dynamical group. As other applications of our general results we give a proof of the spectral theorem for symplectic transformations of classical mechanics, demonstrate the coincidence of the symplectic group $\operatorname{Sp}(2 n)$ with the real subgroup of a matrix group that is isomorphic to the pseudounitary group $\mathrm{U}(n, n)$, and elaborate on an approach to second quantization that makes use of the underlying pseudounitary dynamical groups. (C) 2004 American Institute of Physics. [DOI: 10.1063/1.1646448]
\end{abstract}

\section{INTRODUCTION}

For the past 2 years we have witnessed a growing interest in pseudo-Hermitian Hamiltonians. ${ }^{1-13}$ Initially, the concept of a pseudo-Hermitian operator was developed to describe the mathematical structure of (the possibly nonunitary) $P T$-symmetric quantum systems. ${ }^{1,2}$ Then it became clear that any diagonalizable Hamiltonian that admitted a symmetry generated by an invertible antilinear operator was necessarily pseudo-Hermitian. ${ }^{3,9}$ The intriguing spectral properties of pseudo-Hermitian Hamiltonians generalize to the class of block-diagonalizable Hamiltonians with finite-dimensional blocks, ${ }^{6}$ so does the connection with antilinear symmetries. ${ }^{10}$ Among the most important outcomes of the study of pseudo-Hermitian Hamiltonians is the recent solution of the old problem of constructing invariant positive-definite inner products on the solution space of the Klein-Gordon-type equations. ${ }^{14,15}$

A quantum system with a (time-independent) pseudo-Hermitian Hamiltonian has necessarily a pseudounitary evolution. Pseudounitary quantum systems with a two-dimensional Hilbert space provide the simplest nontrivial examples of such systems. As shown in Ref. 14, a classical simple harmonic oscillator is equivalent to a pseudounitary quantum system with a two-dimensional Hilbert space. Recently Ahmed and Jain ${ }^{11,12}$ and Ahmed $^{13}$ have considered the application of certain $2 \times 2$ pseudo-Hermitian matrices in statistical mechanics and elaborated on the fact that they form a Lie algebra.

The purpose of this paper is threefold. First, we use the method of Ref. 6 to obtain a characterization of the block-diagonalizable pseudounitary operators having finite-dimensional diagonal blocks. Next, we confine our attention to pseudounitary matrices and show that they are obtained by exponentiating pseudo-Hermitian matrices. This is a nontrivial result, because, for a fixed $\eta$, not every $\eta$-pseudounitary matrix is the exponential of $i=\sqrt{-1}$ times an $\eta$-pseudo-Hermitian matrix. Finally, we emphasize that unlike the set of $\eta$-pseudounitary operators (with a fixed $\eta$ ), the

${ }^{a)}$ Electronic mail: amostafazadeh@ku.edu.tr 
set of all pseudounitary operators does not form a group. If the Hilbert space in which these operators act is finite dimensional, then the group of $\eta$-pseudounitary operators is isomorphic to one of the groups $\mathrm{U}(n)$ or $\mathrm{U}(n, m)$ for some $m, n \in \mathbb{Z}^{+}$. For example, the Lie algebra of the pseudounitary matrices constructed in Ref. 11 is isomorphic to $\mathrm{u}(1,1)$. This follows from the fact that the corresponding inner product is indefinite; there is no need to go through the calculation of the structure constants as done in Ref. 11.

The paper is organized as follows. In Sec. II we present a brief discussion of some basic properties of pseudounitary operators and their relevance to symplectic transformations. In Sec. III we explore block-diagonalizable pseudounitary operators with finite-dimensional diagonal blocks. In Sec. IV we use the results of Secs. II and III to study pseudounitary matrices. In Sec. V we offer a thorough discussion of the $2 \times 2$ pseudounitary matrices. In Sec. VI we study an application of our general results for a quantum system with a pseudounitary dynamical group and elaborate on the relation between the choice of the dynamical group and the issue of second quantization. Finally, in Sec. VII we provide a survey of our main results and present our concluding remarks.

\section{PSEUDO-HERMITIAN AND PSEUDOUNITARY OPERATORS}

By definition, ${ }^{1}$ a linear operator $H: \mathcal{H} \rightarrow \mathcal{H}$ acting in a Hilbert space $\mathcal{H}$ is said to be pseudoHermitian if there exists a linear, invertible, Hermitian operator $\eta: \mathcal{H} \rightarrow \mathcal{H}$ such that

$$
H^{\dagger}=\eta H \eta^{-1} \text {. }
$$

For a given pseudo-Hermitian operator $H$, the operator $\eta$ satisfying (1) is not unique.,14 Each choice of $\eta$ determines a possibly indefinite inner product (a pseudoinner product) on $\mathcal{H}$, namely,

$$
\langle\langle\psi, \phi\rangle\rangle_{\eta}:=\langle\psi \mid \eta \phi\rangle,
$$

where $\psi, \phi \in \mathcal{H}$, and $\langle\mid\rangle$ is the original inner product of $\mathcal{H}$. Conversely, every pseudoinner product on $\mathcal{H}$ has the form (2). As a result, $\eta$ is sometimes called a metric operator.

If we make a particular choice for $\eta$, we say that $H$ is $\eta$-pseudo-Hermitian. In this case, it is Hermitian with respect to the inner product $\left\langle\langle,\rangle_{\eta}\right.$. Therefore, the study of $\eta$-pseudo-Hermitian operators is equivalent to the study of Hermitian operators in a vector space with an indefinite metric. ${ }^{16}$ The application of the latter in quantum physics dates back to the 1940 s. ${ }^{17}$ See also Refs. 18 and 19. As emphasized in Ref. 19, there is an important distinction between the concept of pseudo-Hermiticity, where one does not fix the inner product and has the freedom of choosing it, and the well-studied notion of $\eta$-pseudo-Hermiticity.

We can express the defining condition (1) in the form $H^{\#}=H$ where $H^{\#}:=\eta^{-1} H^{\dagger} \eta$ is the $\eta$-pseudoadjoint of $H$. Using the latter one can also define the notion of an $\eta$-pseudounitary operator $U: \mathcal{H} \rightarrow \mathcal{H}$ by requiring that $U$ satisfies $U^{\#}=U^{-1}$.

Definition: A linear invertible operator $U: \mathcal{H} \rightarrow \mathcal{H}$ is said to be pseudounitary if there exists a linear, invertible, Hermitian operator $\eta: \mathcal{H} \rightarrow \mathcal{H}$ such that $U$ is $\eta$-pseudounitary, i.e.,

$$
U^{\dagger}=\eta U^{-1} \eta^{-1} .
$$

Similarly to the case of pseudo-Hermitian operators, $\eta$ is not unique. If we make a choice for $\eta$, we say that $U$ is $\eta$-pseudounitary. In this case it is not difficult to show that $U$ leaves the pseudoinner product $\left\langle\langle,\rangle_{\eta}\right.$ invariant. This is easily seen by writing (3) in the form

$$
U^{\dagger} \eta U=\eta,
$$

and using (2) and (4) to check that

$$
\langle\langle U \psi, U \phi\rangle\rangle_{\eta}=\langle\langle\psi, \phi\rangle\rangle_{\eta}, \quad \forall \psi, \phi \in \mathcal{H} .
$$

Given an $\eta$-pseudo-Hermitian operator $H$ one can construct a one-parameter family of $\eta$-pseudounitary operators, namely $U(t)=e^{-i t H}$ with $t \in \mathbb{R}$. 
Proposition 1: Let $\epsilon \in \mathbb{R}^{+}, t \in(-\epsilon, \epsilon), H: \mathcal{H} \rightarrow \mathcal{H}$ be a $t$-independent linear operator acting in a Hilbert space $\mathcal{H}, U(t):=e^{-i t H}$, and $\eta: \mathcal{H} \rightarrow \mathcal{H}$ be a $t$-independent Hermitian, invertible, linear operator. Then $H$ is $\eta$-pseudo-Hermitian if and only if $U(t)$ is $\eta$-pseudounitary for all $t$ $\in(-\epsilon, \epsilon)$.

Proof: Suppose that $H$ is $\eta$-pseudo-Hermitian, then a direct application of Eq. (1), $U(t)^{\dagger}$ $=e^{i t H^{\dagger}}$ and $U(t)^{-1}=e^{i t H}$, shows that $U(t)$ satisfies (3), i.e., it is $\eta$-pseudounitary. Conversely, let $U(t)$ be $\eta$-pseudounitary for all $t \in(-\epsilon, \epsilon)$. Then substituting $U(t)$ for $U$ in Eq. (3), taking the derivative of both sides with respect to $t$, and setting $t=0$ in the resulting expression, we find that $H$ satisfies (1), i.e., it is $\eta$-pseudo-Hermitian.

Because $U(t)$ may be identified with the evolution operator for a quantum system having $H$ as its Hamiltonian, a quantum system with a time-independent Hamiltonian has a pseudounitary evolution if and only if the Hamiltonian is pseudo-Hermitian. ${ }^{14}$

The one-parameter family $U(t)$ clearly forms an Abelian Lie group under composition. This is indeed a subgroup of the group $\mathcal{U}_{\eta}(\mathcal{H})$ of all $\eta$-pseudounitary operators. The latter forms a group because for any pair $U_{1}, U_{2}: \mathcal{H} \rightarrow \mathcal{H}$ of $\eta$-pseudounitary operators,

$$
\left(U_{1}^{-1} U_{2}\right)^{\dagger}=U_{2}^{\dagger}\left(U_{1}^{\dagger}\right)^{-1}=\eta U_{2}^{-1} \eta^{-1}\left(\eta U_{1}^{-1} \eta^{-1}\right)^{-1}=\eta U_{2}^{-1} \eta^{-1} \eta U_{1} \eta^{-1}=\eta\left(U_{1}^{-1} U_{2}\right)^{-1} \eta^{-1} .
$$

Therefore $\mathcal{U}_{\eta}(\mathcal{H})$ is a subgroup of the group $\operatorname{GL}(\mathcal{H})$ of all invertible linear transformations acting in $\mathcal{H}$. In Ref. 11 , the authors considered this group for the case $\mathcal{H}=\mathbb{C}^{n}$. They call it the pseudounitary group. This terminology is rather misleading as it does not reflect the important fact that a particular choice for $\eta$ has been made. In fact, it is not true that the product of any two pseudounitary operators $V_{1}$ and $V_{2}$ is pseudounitary. This is because they may belong to $\mathcal{U}_{\eta}(\mathcal{H})$ with different $\eta$. This observation calls for a more careful study of the structure of the set $\mathcal{U}(\mathcal{H})$ $:=\cup_{\eta} \mathcal{U}_{\eta}(\mathcal{H})$ of all pseudounitary operators acting in $\mathcal{H}$.

In the remainder of this section we discuss two simple properties of pseudounitary operators that will be of future use.

Proposition 2: Let $\eta_{1}$ be a Hermitian, invertible, linear operator acting in a Hilbert space $\mathcal{H}$, $A: \mathcal{H} \rightarrow \mathcal{H}, U_{1}: \mathcal{H} \rightarrow \mathcal{H}$ be invertible linear operators, $U_{2}:=A^{-1} U_{1} A$ and $\eta_{2}:=A^{\dagger} \eta_{1} A$. Then $U_{1}$ is $\eta_{1}$-pseudounitary if and only if $U_{2}$ is $\eta_{2}$-pseudo-Hermitian.

Proof: First note that the defining condition (3) may be written in the form $U \eta^{-1} U^{\dagger} \eta=I$, where $I$ is the identity operator. Then a simple calculation shows that

$$
U_{2} \eta_{2}^{-1} U_{2}^{\dagger} \eta_{2}=A^{-1} U_{1} A A^{-1} \eta_{1}^{-1} A^{-1 \dagger} A^{\dagger} U_{1} A^{-1 \dagger} A^{\dagger} \eta_{1} A=A^{-1}\left(U_{1} \eta_{1}^{-1} U_{1}^{\dagger} \eta_{1}\right) A .
$$

Therefore, $U_{1} \eta_{1}^{-1} U_{1}^{\dagger} \eta_{1}=I$ if and only if $U_{2} \eta_{2}^{-1} U_{2}^{\dagger} \eta_{2}=I$.

Proposition 3: Let $U_{1}: \mathcal{H} \rightarrow \mathcal{H}$ be a pseudounitary operator acting in a Hilbert space $\mathcal{H}$ and $u$ be an eigenvalue of $U$. Then $1 / u^{*}$ is also an eigenvalue of $U$. In other words, eigenvalues of $U$ are either unimodular $(|u|=1)$ or they come in inverse-complex-conjugate pairs $\left(u, 1 / u^{*}\right)$.

Proof: Let $|u\rangle$ be an eigenvector of $U$ with eigenvalue $u$, i.e., $U|u\rangle=u|u\rangle$. Acting out both sides of (4) on $u^{-1}|u\rangle$, we find $U^{\dagger} \eta|u\rangle=u^{-1} \eta|u\rangle$. Because $\eta$ is invertible, $\eta|u\rangle \neq 0$. This in turn means that $u^{-1}$ is an eigenvalue of $U^{\dagger}$. But the eigenvalues of $U^{\dagger}$ are complex conjugates of those of $U$. Therefore, $u^{-1 *}=1 / u^{*}$ is an eigenvalue of $U$. If $u=1 / u^{*}, u$ is unimodular; otherwise $\left(u, 1 / u^{*}\right)$ is a pair of distinct inverse-complex-conjugate eigenvalues.

As a straightforward application of Proposition 3 , consider the case that $\mathcal{H}=\mathrm{C}^{2 m}$, for some $m \in \mathbb{Z}^{+}$, and endow $\mathrm{C}^{2 m}$ with the metric operator

$$
\eta_{J}:=i J
$$

where $J: \mathbb{C}^{2 m} \rightarrow \mathbb{C}^{2 m}$ has the following matrix representation in the standard orthonormal basis of $\mathrm{C}^{2 m}$ :

$$
J=\left(\begin{array}{cc}
0_{m} & -1_{m} \\
1_{m} & 0_{m}
\end{array}\right)
$$


Here $0_{m}$ and $1_{m}$ are, respectively, the $m \times m$ zero and identity matrices, respectively. According to (6) and (7), the operator $\eta_{J}$ has a Hermitian matrix representation in an orthonormal basis, and $\eta_{J}^{2}=1$. Hence $\eta_{J}$ is indeed a Hermitian invertible (metric) operator acting in $\mathrm{C}^{2 m}$.

Next, observe that the operator $J$ restricted to $\mathbb{R}^{2 m}$ yields the usual symplectic form ${ }^{20}$ on $\mathbb{R}^{2 m}$. The associated symplectic transformations coincide with real $2 m \times 2 m$ matrices $S$ satisfying ${ }^{20}$

$$
S^{t} J S=J
$$

where $S^{t}$ stands for the transpose of $S$. We can view the symplectic transformations $S$ as linear operators acting in $\mathrm{C}^{2 p}$. Then the condition that they admit real matrix representations (in the standard basis) takes the form

$$
T S T=S,
$$

where $T$ is the (time-reversal) operator defined by $\forall \vec{z} \in C^{2 p}, T \vec{z}=\vec{z}^{*}$. Making use of (6) and the fact that $T^{-1}=T$ and $S^{\dagger}=S^{t}$, we can, respectively, express the defining relations (8) and (9) of the symplectic transformations $S$ as

$$
\begin{gathered}
S^{\dagger} \eta_{J} S=\eta_{J}, \\
{[S, T]=0 .}
\end{gathered}
$$

Because $T$ is an antilinear Hermitian invertible operator, according to Theorem 2 of Ref. 3, Eq. (11) implies that $S$ is a pseudo-Hermitian operator. Furthermore, Eq. (10) means that $S$ is in addition a pseudounitary operator.

In view of Proposition 3 and the spectral characterization theorem for pseudo-Hermitian operators (Ref. 1, Theorem 2), the fact that symplectic transformations are both pseudo-Hermitian and pseudounitary leads to the following well-known spectral theorem for symplectic transformations. ${ }^{20}$

Theorem 1: Let $\lambda$ be an eigenvalue of a symplectic transformation $S$, then so are $\lambda^{*}, 1 / \lambda$, and $1 / \lambda^{*}$.

Proof: Because $S$ is pseudounitary $1 / \lambda^{*}$ is an eigenvalue. Because it is pseudo-Hermitian $\lambda^{*}$ and $\left(1 / \lambda^{*}\right)^{*}=1 / \lambda$ are eigenvalues.

\section{BLOCK-DIAGONALIZABLE PSEUDOUNITARY OPERATORS WITH FINITE- DIMENSIONAL DIAGONAL BLOCKS}

Consider an operator $U: \mathcal{H} \rightarrow \mathcal{H}$ acting in a Hilbert space $\mathcal{H}$ and having a discrete spectrum. Then $U$ is said to be block diagonalizable with finite-dimensional diagonal blocks ${ }^{6}$ if it can be expressed in the form

$$
U=\sum_{n} \sum_{a=1}^{d_{n}}\left(u_{n} \sum_{i=1}^{p_{n, a}}\left|\psi_{n}, a, i\right\rangle\left\langle\phi_{n}, a, i\left|+\sum_{i=1}^{p_{n, a}-1}\right| \psi_{n}, a, i\right\rangle\left\langle\phi_{n}, a, i+1\right|\right),
$$

where $n$ is the spectral label, $u_{n}$ are the eigenvalues of $U, d_{n}$ is the geometric multiplicity of $u_{n}$, $a \in\left\{1,2, \ldots, d_{n}\right\}$ is a degeneracy label, $p_{n, a}$ is the dimension of the Jordan block associated with the labels $n$ and $a$ (these are called the Jordan dimensions ${ }^{6}$ ), and $\left\{\left|\psi_{n}, a, i\right\rangle,\left|\phi_{n}, a, i\right\rangle\right\}$ is a complete biorthonormal system satisfying

$$
\left\langle\psi_{n}, a, i \mid \phi_{m}, b, j\right\rangle=\delta_{m n} \delta_{a b} \delta_{i j}, \quad \sum_{n} \sum_{a=1}^{d_{n}} \sum_{i=1}^{p_{n, a}}\left|\psi_{n}, a, i\right\rangle\left\langle\phi_{m}, a, i\right|=1 .
$$

In view of (12) and (13),

$$
U\left|\psi_{n}, a, 1\right\rangle=u_{n}\left|\psi_{n}, a, 1\right\rangle, \quad U^{\dagger}\left|\phi_{n}, a, p_{n, a}\right\rangle=u_{n}^{*}\left|\phi_{n}, a, p_{n, a}\right\rangle,
$$


i.e., $\left|\psi_{n}, a, 1\right\rangle$ are the eigenvectors of $U$ and $\left|\phi_{n}, a, p_{n, a}\right\rangle$ are the eigenvectors of $U^{\dagger}$. Clearly, the eigenvalues of $U^{\dagger}$ are complex conjugates of those of $U$, and if $U$ is invertible the eigenvalues $u_{n}$ do not vanish.

Lemma 1: Let $U: \mathcal{H} \rightarrow \mathcal{H}$ be an invertible operator acting in a Hilbert space $\mathcal{H}$ and $z \in \mathrm{C}$ $-\{0\}$. Then for all $\ell \in \mathbb{Z}^{+}$,

$$
\operatorname{kernel}\left[\left(U^{-1}-z^{-1}\right)^{\ell}\right]=\operatorname{kernel}\left[(U-z)^{\ell}\right] .
$$

Proof: This identity follows by induction over $\ell$. For $\ell=1$, we have

$$
\begin{aligned}
|\xi\rangle \in \operatorname{kernel}\left[U^{-1}-z^{-1}\right] & \Leftrightarrow\left(U^{-1}-z^{-1}\right)|\xi\rangle=0 \\
& \Leftrightarrow z U\left(U^{-1}-z^{-1}\right)|\xi\rangle=0 \Leftrightarrow(z-U)|\xi\rangle=0 \Leftrightarrow|\xi\rangle \in \operatorname{kernel}[U-z],
\end{aligned}
$$

where we have used the fact that $z U$ is an invertible operator. Relations (16) show that (15) holds for $\ell=1$. Now, suppose (15) holds for some $\ell=k \in \mathbb{Z}^{+}$. Then

$$
\begin{aligned}
|\xi\rangle \in \operatorname{kernel}\left[\left(U^{-1}-z^{-1}\right)^{k+1}\right] & \Leftrightarrow\left(U^{-1}-z^{-1}\right)^{k}\left(U^{-1}-z^{-1}\right)|\xi\rangle=0 \\
& \Leftrightarrow\left(U^{-1}-z^{-1}\right)|\xi\rangle \in \operatorname{kernel}\left[\left(U^{-1}-z^{-1}\right)^{k}\right] \\
& \Leftrightarrow\left(U^{-1}-z^{-1}\right)|\xi\rangle \in \operatorname{kernel}\left[(U-z)^{k}\right] \\
& \Leftrightarrow(U-z)^{k}\left(U^{-1}-z^{-1}\right)|\xi\rangle=0 \\
& \Leftrightarrow z U(U-z)^{k}\left(U^{-1}-z^{-1}\right)|\xi\rangle=0 \\
& \Leftrightarrow(U-z)^{k}(z-U)|\xi\rangle=0 \Leftrightarrow|\xi\rangle \in \operatorname{kernel}\left[(U-z)^{k+1}\right] .
\end{aligned}
$$

Therefore, (15) holds for $\ell=k+1$; by induction, it holds for all $\ell \in \mathbb{Z}^{+}$.

Theorem 2: Let $U: \mathcal{H} \rightarrow \mathcal{H}$ be an operator acting in a Hilbert space $\mathcal{H}$ and having a discrete spectrum. Suppose that $U$ is block diagonalizable with finite-dimensional diagonal blocks so that (12) holds. Then $U$ is pseudounitary if and only if the eigenvalues $u_{n}$ of $U$ are either unimodular (i.e., $\left.\left|u_{n}\right|=1\right)$ or they come in inverse-complex-conjugate pairs $\left(u_{n}, 1 / u_{n}^{*}\right)$ and that the geometric multiplicity and the Jordan dimensions for the inverse-complex-conjugate eigenvalues coincide.

Proof: Suppose that $U$ is pseudounitary. Then, according to Proposition 3 the eigenvalues of $U$ are either unimodular or they come in inverse-complex-conjugate pairs. Suppose that $u_{n}$ and

$1 / u_{n}^{*}$ form a pair of distinct inverse-complex-conjugate eigenvalues. In order to show that they have the same geometric multiplicity and Jordan dimensions we prove that for all $\ell \in \mathbb{Z}^{+}$, $\operatorname{kernel}\left(U-u_{n}\right)^{\ell}$ and $\operatorname{kernel}\left(U-1 / u_{n}^{*}\right)^{\ell}$ have the same (finite) dimension. To see this, first note that $U$ and $U^{\dagger}$ have the same Jordan block structure; in view of (12), for all $\ell \in \mathbb{Z}^{+}$, $\operatorname{kernel}(U$ $\left.-u_{n}\right)^{\ell}$ and $\operatorname{kernel}\left(U^{\dagger}-u_{n}^{*}\right)^{\ell}$ have the same (finite) dimension. Hence they are isomorphic as vector spaces. Next, we use the fact that $\eta$ is an invertible operator to establish the isomorphism between $\operatorname{kernel}\left(U^{\dagger}-u_{n}^{*}\right)^{\ell}$ and

$$
\begin{aligned}
\operatorname{kernel}\left[\eta^{-1}\left(U^{\dagger}-u_{n}^{*}\right)^{\ell} \eta\right]=\operatorname{kernel}\left[\left(\eta^{-1} U^{\dagger} \eta-u_{n}^{*}\right)^{\ell}\right] & =\operatorname{kernel}\left[\left(U^{-1}-u_{n}^{*}\right)^{\ell}\right] \\
& =\operatorname{kernel}\left[\left(U-1 / u_{n}^{*}\right)^{\ell}\right] .
\end{aligned}
$$

Here we have made use of the defining relation (3) and the identity (15) of Lemma 1. This completes the proof that for all $\ell \in \mathbb{Z}^{+}, \operatorname{kernel}\left(U-u_{n}\right)^{\ell}$ is isomorphic to $\operatorname{kernel}\left(U-1 / u_{n}^{*}\right)^{\ell}$. Therefore, they have the same (finite) dimension.

Next, suppose that $U$ has unimodular and/or inverse-complex-conjugate pairs of eigenvalues with identical geometric multiplicity and Jordan dimensions. Then $U$ may be expressed as 


$$
\begin{aligned}
U= & \sum_{\nu_{0}} \sum_{a=1}^{d_{\nu_{0}}}\left(u_{\nu_{0}} \sum_{i=1}^{p_{\nu_{0}, a}}\left|\psi_{\nu_{0}}, a, i\right\rangle\left\langle\phi_{\nu_{0}}, a, i\left|+\sum_{i=1}^{p_{\nu_{0}, a}-1}\right| \psi_{\nu_{0}}, a, i\right\rangle\left\langle\phi_{\nu_{0}}, a, i+1\right|\right) \\
& +\sum_{\nu} \sum_{a=1}^{d_{\nu}}\left[\sum_{i=1}^{p_{\nu, a}}\left(u_{\nu}\left|\psi_{\nu+}, a, i\right\rangle\left\langle\phi_{\nu+}, a, i\left|+\frac{1}{u_{\nu}^{*}}\right| \psi_{\nu-}, a, i\right\rangle\left\langle\phi_{\nu-}, a, i\right|\right)\right. \\
& \left.+\sum_{i=1}^{p_{\nu, a}-1}\left(\left|\psi_{\nu+}, a, i\right\rangle\left\langle\phi_{\nu+}, a, i+1|+| \psi_{\nu_{-}}, a, i\right\rangle\left\langle\phi_{\nu-}, a, i+1\right|\right)\right]
\end{aligned}
$$

where we have set $n=\nu_{0}, \nu+$, or $\nu$ - depending on whether $\left|u_{n}\right|=1,\left|u_{n}\right|>1$, or $\left|u_{n}\right|<1$, respectively, and used $\nu$ to denote the common value of $\nu+$ and $\nu-$. In order to show that $U$, as given by (17), is pseudounitary we construct a Hermitian, invertible, linear operator $\eta$ satisfying (3) or equivalently (4). Consider the ansatz

$$
\begin{aligned}
\eta= & \sum_{\nu_{0}} \sum_{a=1}^{d_{\nu_{0}}} \sum_{i, j=1}^{p_{\nu_{0}, a}} z_{\nu_{0}, a, i, j}\left|\phi_{\nu_{0}}, a, i\right\rangle\left\langle\phi_{\nu_{0}}, a, j\right|+\sum_{\nu} \sum_{a=1}^{d_{\nu}} \sum_{i, j=1}^{p_{\nu, a}}\left(\zeta_{\nu, a, i, j}\left|\phi_{\nu-}, a, j\right\rangle\left\langle\phi_{\nu+}, a, i\right|\right. \\
& \left.+\zeta_{\nu, a, i, j}^{*}\left|\phi_{\nu+}, a, i\right\rangle\left\langle\phi_{\nu-}, a, j\right|\right),
\end{aligned}
$$

where $z_{\nu_{0}, a, i, j}$ and $\zeta_{\nu, a, i, j}$ are complex coefficients and

$$
z_{\nu_{0}, a, i, j}^{*}=z_{\nu_{0}, a, j, i}
$$

The latter relation ensures that $\eta$ is Hermitian. Now, impose the condition (4). Substituting (17) and (18) in (4) and using the biorthonormality and completeness relations (13), we find after a quite lengthy calculation that $z_{\nu_{0}, a, i, j}$ and $\zeta_{\nu, a, i, j}$ are solutions of the following equations for $u$ $=u_{\nu_{0}}, p=p_{\nu_{0}, a}$ and $u=u_{\nu}, p=p_{\nu, a}$, respectively,

$$
\begin{gathered}
x_{1, i}=x_{i, 1}=0, \quad \forall i \in\{1,2, \ldots, p-1\}, \\
u x_{i-1, j}+u^{-1} x_{i, j-1}+x_{i-1, j-1}=0, \quad \forall i, j \in\{2, \ldots, p\} .
\end{gathered}
$$

It turns out that these equations have the following exact solution:

$$
x_{i, j}= \begin{cases}0 & \text { for } i+j \leqslant p, \\
\sum_{k=1}^{i+j-p}\left(\begin{array}{c}
i-k-1 \\
p-j-1
\end{array}\right)(-1)^{i-k} u^{p+i-j-k} x_{k, p} \quad \text { for } j<p<i+j,\end{cases}
$$

where for all $r, s \in \mathbb{Z}^{+}$with $r \leqslant s$

$$
\left(\begin{array}{l}
s \\
r
\end{array}\right):=\frac{s !}{r !(s-r) !},
$$

and $x_{k, p}$ with $k \in\{1,2, \ldots, p\}$ are arbitrary complex numbers. We have obtained the solution (22) by a tedious inspection scheme and checked its validity by direct substitution in (21); it clearly satisfies (20). It is important to note that according to (22), $x_{i, j}$ form a $p \times p$ matrix $x$ of the form 


$$
x=\left(\begin{array}{ccccccc}
0 & 0 & 0 & \cdots & 0 & 0 & x_{1, p} \\
0 & 0 & 0 & \cdots & 0 & x_{2, p-1} & x_{2, p} \\
0 & 0 & 0 & \cdots & x_{3, p-2} & x_{3, p-1} & x_{3, p} \\
\vdots & \vdots & \vdots & \vdots \vdots & \vdots & \vdots & \vdots \\
0 & 0 & x_{p-2,3} & \cdots & x_{p-2, p-2} & x_{p-2, p-1} & x_{p-2, p} \\
0 & x_{p-1,2} & x_{p-1,3} & \cdots & x_{p-1, p-2} & x_{p-1, p-1} & x_{p-1, p} \\
x_{p, 1} & x_{p, 2} & x_{p, 3} & \cdots & x_{p, p-2} & x_{p, p-1} & x_{p, p}
\end{array}\right) .
$$

In view of (22) all the entries of $x$ are determined in terms of the entries in the last column. For example, we have

$$
x_{i, p-i+1}=(-1)^{i-1} u^{2(i-1)} x_{1, p}, \quad \forall i \in\{1,2, \ldots, p\} .
$$

Moreover note that the determinant of $x$ is up to a sign the product of the entries (24). Therefore, $x$ is an invertible matrix provided that $x_{1, p} \neq 0$ and $u \neq 0$. Next, consider the case that $u$ is unimodular and seek for the solutions (22) that make $x$ Hermitian, i.e., find solutions for (20) and (21) subject to the condition

$$
x_{i, j}^{*}=x_{j, i} .
$$

Imposing this condition on the solution (22) restricts the choice of the initially free entries, namely $x_{i, p}$. For example, setting $i=p$ and $j=1$ in (22) or alternatively setting $i=p$ in (24), we find $x_{p, 1}=(-1)^{p-1} u^{2(p-1)} x_{1, p}$. Now, using (25) which implies $x_{p, 1}=x_{1, p}^{*}$, we find

$$
x_{1, p}= \pm \sqrt{(-1)^{p-1}} u^{1-p} \rho,
$$

where $\rho=\left|x_{1, p}\right|$ is an arbitrary non-negative real number. A similar analysis shows that the condition (25) leads to similar restrictions on the choices of $x_{i, p}$ with $i>1$. But these restrictions do not lead to any contradictions, i.e., (25) can always be satisfied. Indeed there are infinitely many solutions of the form (22) that fulfill (25). In particular, if we choose $|u|=1$ and $\rho \neq 0$, the matrix $x$ is an invertible Hermitian matrix. Setting $u=u_{\nu_{0}}$, we have a set of solutions $z_{\nu_{0}, a, i, j}$ of (20) and (21) that respect the condition (19) and that the matrices $z_{v_{0}, a}$ formed out of $z_{v_{0}, a, i, j}$ are invertible. Similarly, setting $u=u_{\nu}$ we have a set of solutions $\zeta_{\nu, a, i, j}$ of (20) and (21) such that the matrices $\zeta_{\nu, a}$ formed out of $\zeta_{\nu, a, i, j}$ are also invertible. The existence of these solutions is equivalent to the existence of a linear operator $\eta$ of the form (18) that satisfies (4) and is Hermitian and invertible. The inverse of $\eta$ is given by

$$
\begin{aligned}
\eta^{-1}= & \sum_{\nu_{0}} \sum_{a=1}^{d_{\nu_{0}}} \sum_{i, j=1}^{p_{\nu_{0}, a}} \tilde{z}_{\nu_{0}, a, i, j}\left|\psi_{\nu_{0}}, a, i\right\rangle\left\langle\psi_{\nu_{0}}, a, j\right|+\sum_{\nu} \sum_{a=1}^{d_{\nu}} \sum_{i, j=1}^{p_{\nu, a}}\left(\tilde{\zeta}_{\nu, a, i, j}\left|\psi_{\nu^{-}}, a, j\right\rangle\left\langle\psi_{\nu+}, a, i\right|\right. \\
& \left.+\tilde{\zeta}_{\nu, a, i, j}^{*}\left|\psi_{\nu^{+}}, a, i\right\rangle\left\langle\psi_{\nu_{-}}, a, j\right|\right),
\end{aligned}
$$

where $\tilde{z}_{\nu_{0}, a, i, j}$ are the entries of the matrix $z_{\nu_{0}, a}^{-1}$, and $\tilde{\zeta}_{\nu, a, i, j}$ are those of $\zeta_{\nu, a}^{-1 \dagger}$. One can check by direct calculation that $\eta^{-1} \eta=1$. This completes the proof of the pseudounitarity of $U$.

\section{PSEUDOUNITARY MATRICES}

According to Theorem 2, a square matrix $U$ is pseudounitary if its eigenvalues are either unimodular or they come in inverse-complex-pairs and that geometric multiplicity and the Jordan dimensions of the latter are identical. A direct consequence of this observation is the following.

Proposition 4: Every pseudounitary matrix $U$ has a unimodular determinant, i.e., $|\operatorname{det} U|=1$. 
Proof: This follows from the fact that in the Jordan canonical form of $U$ the nonunimodular entries come is inverse-complex-conjugate pairs $\left(u_{n}, 1 / u_{n}^{*}\right)$. Hence their product which yields $\operatorname{det} U$ is unimodular.

According to this proposition the set $\mathcal{U}\left(\mathbb{C}^{n}\right)$ of all $n \times n$ pseudounitary matrices is a subset of the group

$$
\Sigma L(n, \mathrm{C}):=\{g \in \mathrm{GL}(n, \mathrm{C})|| \operatorname{det} g \mid=1\},
$$

of $n \times n$ matrices with unimodular determinant. We shall call $\Sigma L(n, \mathrm{C})$ the pseudospecial groups. As a subset of $\operatorname{GL}(n, \mathrm{C}), \Sigma L(n, \mathrm{C})$ is the inverse image of the group $\mathrm{U}(1)$ under the homomorphism det:GL $(n, \mathrm{C}) \rightarrow \mathrm{GL}(1, \mathrm{C})$. Therefore, $\Sigma L(n, \mathrm{C})$ is a subgroup of $\mathrm{GL}(n, C)$. In fact, it is not difficult to show that $\Sigma L(n, \mathrm{C})$ is isomorphic to the product group $\mathrm{U}(1) \times \operatorname{SL}(n, \mathrm{C})$. Note however that not every element of the pseudospecial groups is pseudounitary. For example let $g$ be a $2 \times 2$ diagonal matrix with diagonal entries $2 i$ and $-i / 2$. Clearly, $\operatorname{det} g=1 \in \mathrm{U}(1)$, so $g \in \Sigma L(2, \mathrm{C})$. But, $(2 i)^{-1 *}=i / 2 \neq-i / 2$. Hence the eigenvalues $2 i$ and $-i / 2$ are not inverse-complex-conjugates, and $g$ is not pseudounitary. In general, $\mathcal{U}\left(\mathbb{C}^{n}\right)$ is a proper subset of $\Sigma L(n, \mathbb{C})$.

Next, consider the group $\mathcal{U}_{\eta}\left(\mathrm{C}^{n}\right)$ for a fixed Hermitian invertible $n \times n$ matrix $\eta$. We recall Sylvester's law of inertia according to which $\eta$ satisfies

$$
\eta=A^{\dagger} \eta_{p, q} A
$$

where $A$ is some invertible $n \times n$ matrix and $\eta_{p, q}$ is a diagonal matrix of the form

$$
\eta_{p, q}=\operatorname{diag}(-1,-1, \ldots,-1,1,1, \ldots, 1),
$$

which has $p$ negative and $q:=n-p$ positive entries.

Proposition 5: Let $\eta$ be an $n \times n$ Hermitian and invertible matrix. Then the group $\mathcal{U}_{\eta}\left(\mathbb{C}^{n}\right)$ is isomorphic to the pseudounitary group

$$
\mathrm{U}(p, q):=\left\{g \in \mathrm{GL}(n, \mathrm{C}) \mid g^{\dagger} \eta_{p, q} g=\eta_{p, q}\right\}=\mathcal{U}_{\eta_{p, q}}\left(\mathbb{C}^{n}\right)
$$

for some $p \in\{0,1, \ldots, n\}$ and $q:=n-p$. [Note that $\mathrm{U}(0, n)=\mathrm{U}(n)$.]

Proof: Setting $U_{2}=U, \eta_{2}=\eta$, and $\eta_{1}=\eta_{p, q}$ in Proposition 2, we see that $U \in \mathcal{U}_{\eta}\left(\mathbb{C}^{n}\right)$ if and only if $U_{1}:=A U A^{-1} \in U(p, q)$. Hence, $\mathcal{U}_{\eta}\left(\mathbb{C}^{n}\right)=A^{-1} U(p, q) A$. Because the conjugation $i_{A}: \mathrm{GL}(n, \mathrm{C}) \rightarrow \mathrm{GL}(n, \mathbb{C})$ defined by $i_{A}(g):=A^{-1} g A$ is an automorphism of the group $\operatorname{GL}(n, \mathrm{C})$ that maps $\mathcal{U}_{\eta}\left(\mathbb{C}^{n}\right)$ onto $\mathrm{U}(p, q)$, the subgroups $\mathcal{U}_{\eta}\left(\mathbb{C}^{n}\right)$ and $\mathrm{U}(p, q)$ are isomorphic.

According to Proposition 5, the pseudounitary groups $\mathcal{U}_{\eta}\left(\mathrm{C}^{n}\right)$ are isomorphic to and obtained from the classical groups $\mathrm{U}(p, q)$ [or $\mathrm{U}(n)$ ] by conjugation; $\mathcal{U}_{\eta}\left(\mathbb{C}^{n}\right)=A^{-1} U(p, q) A$ for some $A$ $\in \mathrm{GL}(n, \mathrm{C})$. Therefore, the set $\mathcal{U}\left(\mathrm{C}^{n}\right)$ may be viewed as the union of the orbits of the subgroups $\mathrm{U}(p, q)$ under conjugation in $\mathrm{GL}(n, \mathrm{C})$. Obviously these orbits, which according to Proposition 4 lie in the pseudospecial group $\Sigma L(n, \mathbb{C})$, are not disjoint. For example, $e^{i H} \in \mathcal{U}\left(\mathbb{C}^{n}\right)$ belongs to both $\mathcal{U}_{\eta_{1}}\left(\mathbb{C}^{n}\right)$ and $\mathcal{U}_{\eta_{2}}\left(\mathbb{C}^{n}\right)$, if $H$ is both $\eta_{1^{-}}$and $\eta_{2}$-pseudo-Hermitian. The latter holds if and only if $\eta_{2}=A^{\dagger} \eta_{1} A$ for some $A \in \mathrm{GL}(n, \mathrm{C})$ commuting with $H^{7}$

Another simple consequence of Proposition 5 is the following.

Corollary: Let $m \in \mathbb{Z}^{+}$. Then the group $\mathrm{Sp}(2 m)$ of symplectic transformations of $\mathbb{R}^{2 m}$ is isomorphic to the real subgroup of (a matrix group that is isomorphic to) the pseudounitary group $\mathrm{U}(m, m)$.

Proof: According to the argument given above Theorem 1, $\operatorname{Sp}(2 m)$ may be identified with the subgroup of $\mathcal{U}_{\eta_{J}}\left(\mathbb{C}^{2 m}\right)$ consisting of real matrices. It is not difficult to show that the spectrum of $\eta_{J}$ consists of -1 and 1 each with multiplicity $m$. Hence according to Proposition $5, \mathcal{U}_{\eta_{J}}\left(\mathbb{C}^{2 m}\right)$ is isomorphic to $\mathrm{U}(m, m)$, and $\mathrm{Sp}(2 m)$ is isomorphic to the real subgroup of $\mathcal{U}_{\eta_{J}}\left(\mathrm{C}^{2 m}\right)$.

Note also that according to the argument used in the above proof of Theorem 1 and the spectral characterization theorems for pseudo-Hermitian and pseudounitary operators (i.e., Theorem 1 of Ref. 6 and Theorem 2 above), given an eigenvalue $\lambda$ of a symplectic transformation $S$ $\in \operatorname{Sp}(2 m)$, the eigenvalues $\lambda^{*}, 1 / \lambda$, and $1 / \lambda^{*}$ have the same geometric multiplicity and Jordan 
dimensions as $\lambda$. This in particular proves the well-known fact that $S$ has a unit determinant. In particular, $\operatorname{Sp}(2 m)$ may be identified with the real subgroup of (a matrix group that is isomorphic to) $\mathrm{SU}(m, m)$.

Next, we state and prove the following lemma.

Lemma 2: Let $p \in \mathbb{Z}^{+}, E \in \mathrm{C}$, and $h$ be a $p \times p$ matrix of the Jordan form

$$
h=E 1_{p}+a_{p},
$$

where $1_{p}$ is the $p \times p$ identity matrix and $a_{p}$ is the $p \times p$ matrix

$$
a_{p}:=\left(\begin{array}{ccccccc}
0 & 1 & 0 & \cdots & 0 & 0 & 0 \\
0 & 0 & 1 & \cdots & 0 & 0 & 0 \\
\vdots & \vdots & \ddots & \ddots & \vdots & \vdots & \vdots \\
0 & 0 & 0 & \cdots & 0 & 1 & 0 \\
0 & 0 & 0 & \cdots & 0 & 0 & 1 \\
0 & 0 & 0 & \cdots & 0 & 0 & 0
\end{array}\right)
$$

( $a_{p}$ provides an irreducible representation of the annihilation operator for a para-Fermion of order $\left.p-1^{21}\right)$. Then $e^{i h}$ has the following canonical Jordan form:

$$
e^{i E} 1_{p}+a_{p} .
$$

Equivalently, $e^{i E}$ is the unique eigenvalue of $e^{i h}$ with geometric multiplicity 1 and algebraic multiplicity $p$.

Proof: Using the fact that $a_{p}^{p}=0$, we can easily compute

$$
e^{i h}=e^{i E} \sum_{\ell=0}^{p-1} \frac{i^{\ell} a^{\ell}}{\ell !}
$$

This is an upper triangular matrix with a single eigenvalue (namely $e^{i E}$ ) and a single (linearly independent) eigenvector. Therefore its geometric multiplicity is 1 and its algebraic multiplicity is $p$.

Theorem 3: Every pseudounitary matrix $U$ may be expressed as $e^{i H}$ for some pseudoHermitian matrix $H$.

Proof: Let $U$ be an $n \times n$ pseudounitary matrix. Clearly, $U \in \mathrm{GL}(n, \mathrm{C})$. Now, because the exponential map for the group $\operatorname{GL}(n, \mathrm{C})$ is onto, ${ }^{22}$ there is a square matrix $H$ such that $U=e^{i H}$. We can perform a similarity transformation $H \rightarrow \widetilde{H}:=A^{-1} H A$ that maps $H$ into its Jordan canonical form $\tilde{H}$. We then have

$$
U=A e^{i \tilde{H}} A^{-1}
$$

In view of Proposition 2 and Lemma $2, e^{i \tilde{H}}$ is pseudounitary, and its eigenvalues are of the form $e^{i E_{n}}$ where $E_{n}$ are the eigenvalues of $\tilde{H}$. Moreover, the geometric multiplicity and the Jordan dimensions of (the canonical Jordan form of) $e^{i \widetilde{H}}$ coincide with those of $\widetilde{H}$. Now, because $e^{i \tilde{H}}$ is pseudounitary, Theorem 2 implies that the eigenvalues $e^{i E_{n}}$ of $e^{i \tilde{H}}$ are either unimodular or they come in inverse-complex-conjugate pairs with identical geometric multiplicity and Jordan dimensions. First we consider the unimodular eigenvalues which we denote by $e^{i E_{\nu_{0}}}$. Because 1

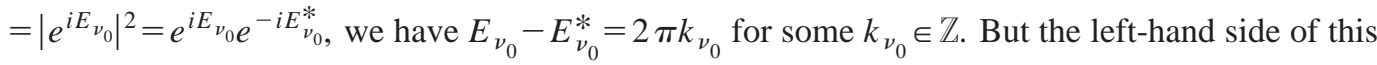
equation is imaginary while its right-hand side is real. This implies $k_{\nu_{0}}=0$. Hence $E_{\nu_{0}}$ is real. Next, consider the eigenvalues $e^{i E_{\nu}}$ that are not unimodular. These are paired with their inverse- 
complex-conjugate, namely, $e^{i E_{\nu}^{*}} \cdot e^{i E_{\nu}}$ and $e^{i E_{\nu}^{*}}$ have the same geometric multiplicity $d_{\nu}$ and Jordan dimensions $p_{\nu, a}$. Because $e^{i E_{v}^{*}}$ is an eigenvalue of $e^{i \widetilde{H}}$, according to Lemma 2 there is an eigenvalue $E_{\nu}^{\prime}$ of $\widetilde{H}$ such that

$$
e^{i E_{\nu}^{*}}=e^{i E_{\nu}^{\prime}}
$$

and that $E_{\nu}^{\prime}$ has the same geometric multiplicity and Jordan dimensions as $e^{i E_{\nu}^{*}}$. Hence the geometric multiplicity and Jordan dimensions of $E_{\nu}^{\prime}$ are, respectively, $d_{\nu}$ and $p_{\nu, a}$. Furthermore, Eq. (35) implies $E_{\nu}^{\prime}=E_{\nu}^{*}+2 \pi k_{\nu}$ for some $k_{\nu} \in \mathbb{Z}$. Now, let $E_{\nu+}$ and $E_{\nu-}$, respectively, denote the eigenvalues of $\widetilde{H}$ with positive and negative imaginary part. In view of the preceding argument, for each $E_{\nu+}$ there is an eigenvalue $E_{\nu-}=E_{\nu+}^{*}+2 \pi k_{\nu+}$. Furthermore all the eigenvalues with negative imaginary part may be obtained from the eigenvalues with positive imaginary part in this way. Now, let $\widetilde{H}^{\prime}$ be the matrix obtained from $\widetilde{H}$ by replacing the eigenvalues $E_{\nu-}$ with $E_{\nu-}^{\prime}$ $:=E_{\nu-}-2 \pi k_{\nu+}=E_{\nu+}^{*}$. Then, by construction, $\widetilde{H}^{\prime}$ has real and/or complex-conjugate pairs of eigenvalues, the latter having identical geometric multiplicity and Jordan dimensions. In light of Theorem 1 of Ref. 6 , this implies that $\widetilde{H}^{\prime}$ is pseudo-Hermitian. One can also check that

$$
e^{i \tilde{H}^{\prime}}=e^{i \tilde{H}} .
$$

Next, let

$$
H^{\prime}:=A \widetilde{H}^{\prime} A^{-1} .
$$

Clearly, $\widetilde{H}^{\prime}$ is the Jordan canonical form of $H^{\prime}$. In particular, $H^{\prime}$ is also pseudo-Hermitian. Combining Eqs. (34), (36), and (37), we finally have

$$
U=A e^{i \tilde{H}^{-1}}=A e^{i \tilde{H}^{\prime}} A^{-1}=e^{i A \tilde{H}^{\prime} A^{-1}}=e^{i H^{\prime}} .
$$

This completes the proof of the fact that $U$ is the exponential of $i$ times a pseudo-Hermitian matrix.

Corollary 1: A square matrix $U$ is pseudounitary if and only if $-i \ln U$ is pseudo-Hermitian, i.e., $U=e^{i H}$ for a pseudo-Hermitian matrix $H$.

Proof: If $U$ is pseudounitary, then according to Theorem 3 it is of the form $e^{i H}$ for some pseudo-Hermitian matrix. If $U=e^{i H}$ for a pseudo-Hermitian matrix $H$, then setting $\epsilon=2, t=-1$ in Proposition 1 we find that $U=\mathrm{U}(-1)$ is pseudounitary.

Corollary 1 is rather surprising, for it is well known that the exponential map is not onto for pseudounitary groups such as $\mathrm{U}(1,1){ }^{22}$ This does not however contradict the statement of Corollary 1 , because when one speaks of a pseudounitary group one fixes the operator $\eta$. What has been done in the proof of Theorem 3 is to show that for a given pseudounitary operator $U$ there is an $\eta$ such that $U$ is $\eta$-pseudounitary and $H:=-\ln U$ is $\eta$-pseudo-Hermitian. This is not equivalent to the erroneous statement that given an $\eta,-i \ln U$ is $\eta$-pseudo-Hermitian for every $\eta$-pseudounitary matrix $U$. The exponential map for the pseudounitary group $\mathcal{U}_{\eta}\left(\mathrm{C}^{n}\right)$ is generally not onto, but the exponential map for the set of all pseudounitary matrices is onto. This is another demonstration of the importance of the difference between the notions of $\eta$-pseudo-Hermiticity (respectively, $\eta$-pseudounitarity) and pseudo-Hermiticity (respectively, pseudounitarity). ${ }^{19}$

\section{2×2 PSEUDOUNITARY MATRICES}

In this section we shall study the case $n=2$ in more detail. The following corollary of Theorem 3 yields the general form of $2 \times 2$ pseudounitary matrices.

Corollary 2: A $2 \times 2$ matrix $U$ is pseudo-Hermitian if and only if $U=A^{-1} D A$ where $A$ is an invertible $2 \times 2$ matrix and $D$ is a matrix assuming one of the following three forms: 


$$
\begin{gathered}
D_{1}=\left(\begin{array}{cc}
e^{i \theta} & 0 \\
0 & e^{i(\varphi-\theta)}
\end{array}\right), \quad \theta, \varphi \in \mathbb{R}, \\
D_{2}=\left(\begin{array}{cc}
r e^{i \theta} & 0 \\
0 & e^{i \theta} / r
\end{array}\right), \quad r \in \mathbb{R}^{+}, \quad \theta \in \mathbb{R}, \\
D_{3}=\left(\begin{array}{cc}
e^{i \theta} & 1 \\
0 & e^{i \theta}
\end{array}\right), \quad \theta \in \mathbb{R} .
\end{gathered}
$$

Proof: Block diagonalizing $U$ we find a matrix $D$ which is either diagonal or has the form

$$
D=\left(\begin{array}{ll}
u & 1 \\
0 & u
\end{array}\right)
$$

where $u \in$ C. According to Proposition 2, $D$ is also pseudounitary. This together with Theorem 3 imply that

(i) if $D$ is diagonal, its eigenvalues are either both unimodular, i.e., $D$ is of the form (38), or they are inverse-complex-conjugate, i.e., $D$ is of the form (39);

(ii) if $D$ has the form (41), then it has a single eigenvalue $u$ which is necessarily unimodular. That is $D$ is of the form (40).

In order to demonstrate the utility of Theorem 3, here we include a direct proof of Corollary 2. This proof involves the calculation of the matrices $\eta$ whose general form is given in the proof of Theorem 2.

A direct proof of corollary 2: First consider the case that $U$ is diagonalizable, then the canonical Jordan form $D=A U A^{-1}$ of $U$ is diagonal. Clearly $\operatorname{det} D=\operatorname{det} U$ and according to Proposition $4 \operatorname{det} U \in \mathrm{U}(1)$. Hence $|\operatorname{det} D|=1$. This implies that $D$ must have the form

$$
D=\left(\begin{array}{cc}
\zeta & 0 \\
0 & e^{i \varphi} / \zeta
\end{array}\right)
$$

where $\zeta:=r e^{i \theta} \in \mathrm{C}-\{0\}$ and $e^{i \varphi} \in U(1)$, i.e., $r \in \mathbb{R}^{+}$and $\theta, \varphi \in \mathbb{R}$. Next, note that in view of Proposition 2, $U$ is $\eta$-pseudounitary if and only if $D$ is $A^{-1 \dagger} \eta A^{-1}$-pseudounitary. This reduces the problem to finding the necessary and sufficient conditions on $\zeta$ (alternatively $r, \theta$ ) and $\varphi$ that make $D$ pseudounitary. Using the general form

$$
\eta=\left(\begin{array}{cc}
a & \xi \\
\xi^{*} & b
\end{array}\right), \quad a, b \in \mathbb{R}, \quad \xi \in \mathrm{C}, \quad a b \neq|\xi|^{2}
$$

of the Hermitian matrix $\eta$ and the fact that $D$ is $\eta$-pseudounitary for some $\eta$ of the form (43), i.e., $D^{\dagger}=\eta D^{-1} \eta^{-1}$ or $D^{\dagger} \eta D=\eta$, we find that for $\xi=0: r=1$ and $D=D_{1}$, and for $\xi \neq 0: e^{i \varphi}=e^{i \theta}$ and $D=D_{2}$. Next, consider the case that $U$ is not diagonalizable. Then $D$ has the form (41). Again because $D$ is pseudounitary, $\operatorname{det} D \in \mathrm{U}(1)$. This implies $u \in \mathrm{U}(1)$, i.e., $u=e^{i \theta}$ for some $\theta \in \mathbb{R}$. Substituting this expression and the general form (43) of $\eta$ in $D^{\dagger} \eta D=\eta$, we find that this equation can always be satisfied without restricting $\theta$. Therefore, in this case $D=D_{3}$.

The above analysis also yields the form of $\eta$ for each of the cases considered.

(1) For $D=D_{1}$, there are two possibilities.

(1a) $e^{i \varphi} \neq e^{2 i \theta}$ : In this case, $\xi=0$ and $\eta$ has the diagonal form

$$
\eta=\eta_{1}:=\left(\begin{array}{ll}
a & 0 \\
0 & b
\end{array}\right), \quad a, b \in \mathbb{R}-\{0\} .
$$


TABLE I. Operators $H_{D}$ and $\eta$ for $D=D_{1}, D_{2}, D_{3}$.

\begin{tabular}{|c|c|c|c|c|}
\hline$i$ & $D_{i}$ & $H_{D_{i}}$ & & $\eta_{i}$ \\
\hline 1 & $\left(\begin{array}{cc}e^{i \theta} & 0 \\
0 & e^{i(\varphi-\theta)}\end{array}\right)$ & $\left(\begin{array}{cc}\theta & 0 \\
0 & \varphi-\theta\end{array}\right)$ & $\left(\begin{array}{l}a \\
0\end{array}\right.$ & $\left.\begin{array}{l}0 \\
b\end{array}\right)$ \\
\hline 2 & $\left(\begin{array}{cc}r e^{i \theta} & 0 \\
0 & e^{i \theta / r}\end{array}\right)$ & $\left(\begin{array}{cc}\theta-i \ln r & 0 \\
0 & \theta+i \ln r\end{array}\right)$ & $\left(\begin{array}{l}0 \\
\xi^{*}\end{array}\right.$ & $\left.\begin{array}{l}\xi \\
0\end{array}\right)$ \\
\hline 3 & $\left(\begin{array}{cc}e^{i \theta} & 1 \\
0 & e^{i \theta}\end{array}\right)$ & $\left(\begin{array}{cc}\theta & \theta\left(e^{i \theta}-1\right)^{-1} \\
0 & \theta\end{array}\right)$ & $\left(\begin{array}{c}0 \\
\mp i r e^{i \theta}\end{array}\right.$ & $\left.\begin{array}{c} \pm i r e^{-i \theta} \\
0\end{array}\right)$ \\
\hline
\end{tabular}

Because $a$ and $b$ may have arbitrary sign, the group $\mathcal{U}_{\eta_{1}}\left(C^{2}\right)$ is isomorphic to either $\mathrm{U}(2)$ or $\mathrm{U}(1,1)$.

(1b) $e^{i \varphi}=e^{2 i \theta}$ : In this case, $D=e^{i \theta} I$ where $I$ is the $2 \times 2$ unit matrix. Hence, there is no restriction on $\eta$; it has the general form (43), and $\mathcal{U}_{\eta}\left(\mathrm{C}^{2}\right)$ is isomorphic to either $\mathrm{U}(2)$ or $\mathrm{U}(1,1)$. (2) If $D=D_{2}$ with $r=1$ we recover the case (1b). If $D=D_{2}$ and $r \neq 1$, then $a=b=0$ and $\eta$ has the off-diagonal form

$$
\eta=\eta_{2}:=\left(\begin{array}{cc}
0 & \xi \\
\xi^{*} & 0
\end{array}\right), \quad \xi \in \mathrm{C}-\{0\}
$$

Because $\eta_{2}$ is an indefinite matrix, $\mathcal{U}_{\eta_{2}}\left(\mathrm{C}^{2}\right)$ is isomorphic to $\mathrm{U}(1,1)$. (3)

(3) If $D=D_{3}$. Then $\eta$ has the general form

$$
\eta=\eta_{3}:=\left(\begin{array}{cc}
0 & \pm i r e^{-i \theta} \\
\mp i r e^{i \theta} & 0
\end{array}\right), \quad r \in \mathbb{R}^{+}, \quad \theta \in \mathbb{R},
$$

and $\mathcal{U}_{\eta_{3}}\left(\mathrm{C}^{2}\right)$ is isomorphic to $\mathrm{U}(1,1)$.

We can check that the above expressions for $\eta$ are consistent with the general form of $\eta$ as given in the proof of Theorem 2. Furthermore, we can obtain the explicit form of the operator $H:=-i \ln U$. In view of the identity $U=A^{-1} D A$, it is not difficult to see that if we obtain an operator $H_{D}$ satisfying $D=e^{i H_{D}}$, then $H=A^{-1} H_{D} A$ will satisfy $U=e^{i H}$. Table I gives the operators $H_{D}$ and $\eta$ for $D=D_{1}, D_{2}, D_{3}$. Note that in this table $\theta, \varphi \in \mathbb{R}, r \in \mathbb{R}^{+}, a, b \in \mathbb{R}-\{0\}$, $\xi \in \mathrm{C}-\{0\}$, and that the trivial case where $D$ is proportional to the unit matrix is omitted.

\section{PSEUDOUNITARY DYNAMICAL GROUPS AND THE HARMONIC OSCILLATOR}

Suppose that $H$ is a $2 \times 2$ pseudo-Hermitian matrix serving as the (time-independent) Hamiltonian for a quantum system, $U(t):=e^{-i t H}$ is the corresponding evolution operator, $\mathcal{E}_{H}$ is the set of all invertible Hermitian $2 \times 2$ matrices $\eta$ satisfying (1), and

$$
\mathcal{U}_{H}:=\bigcup_{\eta \in \mathcal{E}_{H}} \mathcal{U}_{\eta}\left(\mathrm{C}^{2}\right), \quad \mathcal{G} \mathcal{U}_{H}:=\bigcup_{\eta \in \mathcal{E}_{H}} \mathcal{G} \mathcal{U}_{\eta}\left(\mathrm{C}^{2}\right),
$$

where $\mathcal{G U}_{\eta}\left(\mathrm{C}^{2}\right)$ denotes the Lie algebra of $\mathcal{U}_{\eta}\left(\mathrm{C}^{2}\right)$. Then clearly $i H \in \mathcal{G U}_{H}$ and for all $t \in \mathbb{R} U(t)$ $\in \mathcal{U}_{H}$. This in particular means that for each $\eta \in \mathcal{E}_{H}, \mathcal{U}_{\eta}\left(\mathrm{C}^{2}\right)$ serves as a dynamical group for the quantum system. ${ }^{23}$ If $H$ is diagonalizable with a real spectrum then the dynamical group may be taken to be (isomorphic to) either $\mathrm{U}(2)$ or $\mathrm{U}(1,1)$ (or one of their subgroups). If $H$ has (nonreal) complex eigenvalues or if it is not diagonalizable, then the dynamical group is necessarily (isomorphic to a subgroup of) $\mathrm{U}(1,1)$. [The generalization of this statement to arbitrary block- 
diagonalizable pseudo-Hermitian Hamiltonians with finite-dimensional blocks is immediate. If the Hamiltonian is not diagonalizable or has complex eigenvalues, then the dynamical groups that the system admits are necessarily (isomorphic to a subgroup of) $\mathrm{U}(p, q)$ with $p \neq 0 \neq q$.]

A concrete example is provided by the classical equation of motion for a simple harmonic oscillator of frequency $\omega$,

$$
\ddot{x}+\omega^{2} x=0 .
$$

As explained in Refs. 4 and 14, this equation is equivalent to the Schrödinger equation,

$$
i \hbar \frac{d}{\mathrm{~d} t} \Psi=H \Psi
$$

where

$$
\Psi=:\left(\begin{array}{c}
x+i \lambda \dot{x} \\
x-i \lambda \dot{x}
\end{array}\right), \quad H=: \frac{\hbar}{2}\left(\begin{array}{cc}
\lambda \omega^{2}+\lambda^{-1} & \lambda \omega^{2}-\lambda^{-1} \\
-\lambda \omega^{2}+\lambda^{-1} & -\lambda \omega^{2}-\lambda^{-1}
\end{array}\right),
$$

and $\lambda \in \mathbb{R}^{+}$is a time scale. Clearly $H$ is a traceless matrix. It is also easy to check that $\operatorname{det} H$ $\in \mathbb{R}$ if and only if $\omega^{2} \in \mathbb{R}$. Therefore, according to Theorem 3 of Ref. 6, $H$ is a pseudo-Hermitian matrix provided that $\omega^{2} \in \mathbb{R}$. Furthermore, $H$ is diagonalizable unless $\omega=0$.

In the following we shall only consider the case $\omega^{2} \in R$.

For $\omega \neq 0$, we can easily solve the eigenvalue problem and diagonalize $H$. The corresponding diagonal matrix has the form $H_{D}=\hbar \omega \sigma_{3}$ where $\sigma_{3}$ is the diagonal Pauli matrix $\operatorname{diag}(1,-1)$. Comparing the expression for $H_{D}$ with the results given in the above table, we see that $H_{D}$ is $\eta$-pseudo-Hermitian with respect to a diagonal metric operator $\eta$ of the form (44) provided that $\omega^{2}>0$. In this case the system admits both the dynamical groups $\mathrm{U}(2)$ and $\mathrm{U}(1,1)$. If $\omega^{2}<0, H$ is $\eta$-pseudo-Hermitian with respect to an off-diagonal metric operator $\eta$ of the form (45) and the system only admits the dynamical group $\mathrm{U}(1,1)$. Finally for $\omega=0, H$ is not diagonalizable; $U$ $=e^{i H}$ has the Jordan canonical form $D_{3}$; it is $\eta$-pseudounitary for a metric operator $\eta$ of the form (46) and the system admits the dynamical group $\mathrm{U}(1,1)$. [It is interesting to observe that the noncompact dynamical group $\mathrm{U}(1,1)$ arises for the case that $\omega^{2}<0$ where Eq. (47) admits unbounded solutions.]

For the case $\omega^{2}>0$, the freedom in the choice of the dynamical group is equivalent to the choice of a positive-definite or an indefinite inner product on the space of solutions of Eq. (47). ${ }^{14}$ This freedom does not exist if $\omega^{2} \leqslant 0$.

Now, consider changing the parameter $\omega^{2}$ from a positive value down to a negative value. If one adopts an indefinite (but possibly $\omega^{2}$-dependent) inner product, one can keep $H$ Hermitian with respect to this inner product and view the evolution operator as tracing a curve in the dynamical group $\mathrm{U}(1,1)$. The best-known example is the Klein-Gordon inner product that corresponds to the choice $\eta=\sigma_{3}$, and therefore is independent of the value of the parameter $\omega^{2}$. However, if one initially adopts a (possibly $\omega^{2}$-dependent) positive-definite inner product, one cannot maintain the Hermiticity of $H$ with respect to this inner product once $\omega^{2}$ crosses zero. The dynamical group undergoes an abrupt transition from the group $\mathrm{U}(2)$ to the group $\mathrm{U}(1,1)$. This transition may be identified with the change of the signature of the metric (operator).

For $\omega^{2}>0$, one may endow the Hilbert space $\left(\mathrm{C}^{2}\right)$ with a positive-definite invariant inner product. In this case the system has a $\mathrm{U}(2)$ dynamical group and is physically equivalent to the two-level spin system ${ }^{23}$ i.e., a spin-1/2 particle interacting with a fixed magnetic field. The time evolution is clearly unitary. This equivalence is destroyed once $\omega^{2}$ becomes nonpositive. In this case the dynamical group is $\mathrm{U}(1,1)$ and the system does not admit a unitary evolution with respect to any positive-definite inner product on $\mathbb{C}^{2}$. For the case that $\omega^{2}>0$ one could as well choose an indefinite invariant inner product (this is precisely what was done historically). But such a choice leads to a nonunitary quantum system with a two-dimensional Hilbert space and a U(1,1) dynamical group. As is well known the corresponding quantum harmonic oscillator also has a $\mathrm{U}(1,1)$ [or 
rather $\mathrm{SU}(1,1)]$ dynamical group. ${ }^{23}$ Therefore, as far as the dynamics is concerned the nonunitary system describing the dynamics of the classical oscillator is equivalent to the unitary quantum harmonic oscillator.

For the case $\omega^{2}>0$ there are therefore two alternatives. One is to choose a positive-definite invariant inner product which corresponds to the dynamical group $\mathrm{U}(2)$. The other is to choose an indefinite invariant inner product which leads to the dynamical group $\mathrm{U}(1,1)$.

Now, suppose that one wishes to keep the same dynamical group but insists on being able to describe the dynamics using a unitary quantum system. In the first alternative this is already the case. But in the second alternative one needs to use an infinite-dimensional Hilbert space, because being a noncompact Lie group $\mathrm{U}(1,1)$ does not admit a finite-dimensional unitary representation. Therefore it is the demand for unitarity that leads to the quantization of the oscillator. The latter is however not unique because $\mathrm{U}(1,1)$ has inequivalent unitary irreducible representations. This does not lead to any problems, because the dynamics always takes place in the dynamical group. ${ }^{23}$ As a result the dynamical aspects of all possible quantum systems associated with the classical harmonic oscillator are equivalent. [Note that here quantization does not mean the canonical quantization which is unique in the sense that the Weyl-Heisenberg algebra has a unique irreducible (projective) representation. It means defining the Hilbert space as the representation space of a unitary irreducible projective representation of the dynamical group, and representing the Hamiltonian as an element of the Lie algebra of the dynamical group.]

The above two alternatives are also available in describing free Klein-Gordon fields (or more generally Klein-Gordon fields interacting with a stationary magnetic field). The second alternative applies more generally even to the cases of interacting fields. It corresponds to Dirac's method of second quantization that forms the foundations of quantum field theories. The first alternative was noticed quite recently. ${ }^{14,15,24}$ (See however Ref. 25 that were brought to the author's attention after the completion of this project.) Its advantage is to provide a genuine probability interpretation for first quantized Klein-Gordon fields. ${ }^{26}$ Its main application is in quantum cosmology. ${ }^{15}$

\section{CONCLUSION}

In this paper, we explored various properties of pseudounitary operators and proved a spectral characterization theorem for the class of block-diagonalizable pseudounitary operators with finitedimensional blocks. We applied our results to clarify the structure of pseudounitary matrices paying attention to the role of the inner product and the fact that it is not unique. We showed that the relationship between Hermitian and unitary matrices generalize to the pseudo-Hermitian and pseudounitary matrices. Specifically every pseudounitary matrix is the exponential of $i$ times a pseudo-Hermitian matrix.

We showed that the symplectic transformations of classical mechanics are certain pseudounitary and pseudo-Hermitian operators. This led to a proof of the spectral theorem for symplectic matrices and to the identification of the symplectic groups $\operatorname{Sp}(2 n)$ with the real subgroups of certain pseudounitary matrix groups that are isomorphic to $\mathrm{U}(n, n)$. The description of the symplectic transformations in terms of pseudounitary and pseudo-Hermitian operators suggests the possibility of the application of the latter in classical mechanics. (For a related discussion see Ref. 27.)

Furthermore, we derived the canonical forms of arbitrary $2 \times 2$ pseudounitary matrices, and studied the pseudounitary system describing a classical harmonic oscillator. For real nonzero frequencies, this system admits both the dynamical groups $\mathrm{U}(2)$ or $\mathrm{U}(1,1)$. If one imposes the condition of the unitarity of the evolution, then the choice $\mathrm{U}(2)$ identifies the dynamics of the oscillator with that of a two-level quantum system, and the choice $\mathrm{U}(1,1)$ leads to a quantization of the oscillator. This picture provides a rather interesting link between the demand for unitarity and the need for (second) quantization.

\section{ACKNOWLEDGMENT}

This work has been supported by the Turkish Academy of Sciences in the framework of the Young Researcher Award Program (EA-TÜBA-GEBIP/2001-1-1). 
${ }^{1}$ A. Mostafazadeh, J. Math. Phys. 43, 205 (2002).

${ }^{2}$ A. Mostafazadeh, J. Math. Phys. 43, 2814 (2002).

${ }^{3}$ A. Mostafazadeh, J. Math. Phys. 43, 3944 (2002)

${ }^{4}$ A. Mostafazadeh, Nucl. Phys. B 640, 419 (2002).

${ }^{5}$ A. Mostafazadeh, Mod. Phys. Lett. A 17, 1973 (2002).

${ }^{6}$ A. Mostafazadeh, J. Math. Phys. 43, 6343 (2002); 44, 943(E) (2003).

${ }^{7}$ A. Mostafazadeh, J. Math. Phys. 44, 974 (2003).

${ }^{8}$ Z. Ahmed, Phys. Lett. A 290, 19 (2001); 294, 287 (2002); C.-S. Jia, X.-L. Zeng, and L.-T. Sun, ibid. 294, 185 (2002); C.-S. Jia, P.-Y. Lin, and L.-T. Sun, ibid. 298, 78 (2002); C.-S. Jia, S.-C. Li, Y. Li, and L.-T. Sun, ibid. 300, 115 (2002); B. Bagchi and C. Quesne, ibid. 301, 173 (2002); G. Scolarici and L. Solombrino, ibid. 303, 239 (2002); C.-S. Jia, Y. Sun, and Y. Li, ibid. 305, 231 (2002); G. Scolarici, J. Phys. A 35, 7493 (2002); R. N. Deb, A. Khare, and B. D. Roy, Phys. Lett. A 307, 215 (2003); F. Cannata, M. V. Ioffe, and D. N. Nishnianidze, ibid. 310, 344 (2003).

${ }^{9}$ L. Solombrino, J. Math. Phys. 43, 5439 (2002).

${ }^{10}$ G. Scolarici and L. Solombrino, J. Math. Phys. 44, 4450 (2003).

${ }^{11}$ Z. Ahmed and S. R. Jain, Phys. Rev. E 67, 045106 (2003).

${ }^{12}$ Z. Ahmed and S. R. Jain, J. Phys. A 36, 9711 (2003).

${ }^{13}$ Z. Ahmed, Phys. Lett. A 310, 139 (2003).

${ }^{14}$ A. Mostafazadeh, Class. Quantum Grav. 20, 155 (2003).

${ }^{15}$ A. Mostafazadeh, Ann. Phys. (N.Y.) 309, 1 (2004).

${ }^{16}$ J. Bognár, Indefinite Inner Product Spaces (Springer, Berlin, 1974); T. Ya. Azizov and I. S. Iokhvidov, Linear Operators in Spaces with Indefinite Metric (Wiley, Chichester, 1989).

${ }^{17}$ W. Pauli, Rev. Mod. Phys. 15, 175 (1943).

${ }^{18}$ A. Ramírez and B. Mielnik, quant-ph/0211048.

${ }^{19}$ A. Mostafazadeh, Czech. J. Phys. 53, 1079 (2003).

${ }^{20}$ V. I. Arnold, Mathematical Methods of Classical Mechanics (Springer, New York, 1989).

${ }^{21}$ Y. Ohnuki and S. Kamefuchi, Quantum Field Theory and Parastatistics (Springer, Berlin, 1982).

${ }^{22}$ J. J. Duistermaat and J. A. Kolk, Lie Groups (Springer, Berlin, 2000); V. S. Varadarajan, Lie Groups, Lie Algebras, and Their Representations (Springer, New York, 1974).

${ }^{23}$ A. Mostafazadeh, Dynamical Invariants, Adiabatic Approximation, and the Geometric Phase (Nova Science Publishers, New York, 2001).

${ }^{24} \mathrm{~A}$. Mostafazadeh, quant-ph/0307059.

${ }^{25}$ J. J. Halliwell and M. E. Ortiz, Phys. Rev. D 48, 748 (1993); P. P. Woodard, Class. Quantum Grav. 10, 483 (1993); J. B. Hartle and D. Marolf, Phys. Rev. D 56, 6247 (1997); F. S. G. Von Zuben, J. Math. Phys. 41, 6093 (2000); J. J. Halliwell and J. Thorwart, Phys. Rev. D 64, 124018 (2001).

${ }^{26}$ A. Mostafazadeh and F. Zamani, quant-ph/0312078.

${ }^{27}$ F. Ventriglia, Mod. Phys. Lett. A 17, 1589 (2002). 